\title{
Berufliche Kompetenzen von Geflüchteten erkennen? Exemplarische Befunde zur Kompetenzmessung im Bereich der Metallbearbeitung und Metallverarbeitung
}

\author{
Andreas Fischer $\cdot$ Kristin Hecker $\cdot$ Iris Pfeiffer
}

Eingegangen: 28. Juni 2018 / Angenommen: 14. Dezember 2018 / Online publiziert: 11. Januar 2019

(C) Der/die Autor(en) 2019

Zusammenfassung Der IdA KompetenzCheck stellt ein wissenschaftlich entwickeltes Testverfahren dar, um non-formal und informell erworbene Kompetenzen sichtbar zu machen, und in Bezug auf deutsche Referenzberufe auszuwerten. Im Rahmen des IdA KompetenzCheck werden Testpersonen mit typischen Handlungsfeldern eines Berufsbildes konfrontiert. Zu jedem Handlungsfeld wird eine Reihe von handlungsrelevanten Fragen präsentiert, deren Beantwortung Rückschlüsse auf die Ausprägung der Kompetenz im jeweiligen Handlungsfeld ermöglicht. Auf diese Weise erlaubt es der IdA KompetenzCheck für ausgewählte deutsche Referenzberufe aus vier Branchen (Metall, Elektro, Logistik sowie Garten- \& Landschaftsbau), die Kompetenzen der Testteilnehmenden zu erfassen.

Im vorliegenden Beitrag wird am Beispiel des IdA KompetenzCheck Metall vorgestellt, wie berufliche Kompetenzen effektiv und effizient über papier- oder computerbasierte Testverfahren getestet werden können und welche Besonderheiten bei der Kompetenzfeststellung von Geflüchteten zu beachten sind. Außerdem werden erstmals empirische Belege für die Zuverlässigkeit und Gültigkeit derartiger Testverfahren erbracht: Anhand einer Stichprobe von 162 Geflüchteten, die eine papierbasierte Fassung des Tests bearbeiteten, wird gezeigt, dass sich mit dem IdA KompetenzCheck reliable und valide Ergebnisse erzielen lassen, die durchweg positiv mit Berufserfahrung korrelieren.

Die Ergebnisse zu diesem und ähnlichen Testverfahren werden kritisch diskutiert und sowohl im Spannungsfeld zwischen Praktikabilität, Reliabilität und Validität,

\footnotetext{
A. Fischer $(\bowtie) \cdot$ K. Hecker $\cdot$ I. Pfeiffer

Forschungsinstitut Betriebliche Bildung (f-bb) gGmbH, Nürnberg, Deutschland

E-Mail: andreas.fischer@f-bb.de

K. Hecker

E-Mail: kristin.hecker@f-bb.de

I. Pfeiffer

E-Mail: iris.pfeiffer@f-bb.de
} 
als auch in Bezug auf andere Verfahren der Kompetenzfeststellung eingeordnet. Zusammenfassend halten wir den IdA KompetenzCheck für einen gelungenen Beitrag zu einer Validierung von ,learning outcomes“ nicht-zertifizierten non-formalen und informellen Lernens, der jedoch im Sinne einer ganzheitlichen Beurteilung von beruflicher Handlungskompetenz andere Verfahren der Kompetenzfeststellung nicht ersetzen, sondern nur ergänzen kann.

Schlüsselwörter Kompetenz · Kompetenztest · Kompetenzfeststellung · Berufserfahrung $\cdot$ Geflüchtete

\section{Recognizing occupational competences of refugees? Exemplary findings on competence measurement in the field of metal working and metal processing}

Abstract The IdA KompetenzCheck represents a scientifically developed test procedure for measuring non-formally and informally acquired competences and for evaluating competences in relation to German occupations. As part of the IdA KompetenzCheck, testees are confronted with an occupational profile's typical fields of action. Multiple action-oriented questions concerning each field of action are presented in order to determine the corresponding level of competence. The IdA KompetenzCheck allows for testing competences of testees with regard to selected German reference occupations from four sectors (Metal, Electrical, Logistics and Gardening \& Landscaping). This article uses the example of the IdA KompetenzCheck Metal to demonstrate how occupational competences can effectively and efficiently be tested via paper or computer-based test procedures and which special features are to be considered when measuring the competence of refugees.

For the first time empirical evidence for the reliability and validity of such test procedures is provided: Based on a sample of 162 refugees, who completed a paper-based version of the test, it is shown that the IdA KompetenzCheck allows for reliable and valid conclusions about occupational competences, and that test scores positively correlate with work experience.

The results of the IdA KompetenzCheck and similar tests are discussed critically and in the field of tension between practicability, reliability and validity, as well as in relation to other competence assessment procedures. In summary, we consider the IdA KompetenzCheck a successful contribution to validate "learning outcomes" of non-certified non-formal and informal learning, that can complement (but not replace) other methods of competence assessment in order to achieve a holistic assessment of occupational competence.

Keywords Competence $\cdot$ Testing - Assessment $\cdot$ Work experience $\cdot$ Refugees 


\section{Stand der Kompetenzen von Geflüchteten auf dem deutschen Arbeitsmarkt}

Seit Anfang 2015 sind mehr als 1,3 Mio. ${ }^{1}$ Asylsuchende nach Deutschland gekommen, rund zwei Drittel aus Syrien, Afghanistan und Irak. Viele dieser Menschen können aufgrund einer positiven Asyl-Entscheidung in Deutschland bleiben, d.h. sie gelten als anerkannte Geflüchtete oder erhalten einen anderen Schutzstatus (BAMF 2016, 2017). Um mehr über den Bildungshintergrund der Asylsuchenden zu erfahren, wurden bereits im September 2016 im Rahmen einer repräsentativen IABBAMF-SOEP-Befragung 2349 Geflüchtete interviewt. ${ }^{2}$ Rund 13\% der Befragten gaben an, einen Hochschulabschluss und 6\% einen beruflichen Abschluss zu besitzen. Im Vergleich zu deutschen Staatsbürgerinnen und Staatsbürgern - hier hatten $21 \%$ einen Hochschulabschluss und 59\% einen beruflichen Abschluss - ist das formale Bildungsniveau geringer. Dies schließt jedoch nicht aus, dass berufliche Fähigkeiten, Kenntnisse und Fertigkeiten vorhanden sind, die z. B. durch On-the-jobTraining oder andere Arbeitserfahrungen erworben wurden und von Arbeitgebern in Deutschland nachgefragt sind.

Die meisten dieser Länder kennen kein Ausbildungssystem, das mit dem deutschen Berufsbildungssystem vergleichbar wäre. Viele handwerkliche, technische und kaufmännische Berufe werden deshalb in den Herkunftsländern ohne formelle Ausbildung ausgeübt. (Brücker et al. 2016, S. 6-7)

Von den befragten Personen gaben $73 \%$ an, bereits vor der Einreise nach Deutschland Berufserfahrungen gesammelt zu haben, davon rund $45 \%$ als Arbeiterinnen und Arbeiter oder Angestellte ohne Führungsposition, 13\% als Angestellte in Führungsposition und $27 \%$ als Selbstständige (von Gostomski et al. 2016, S. 7). Im Durchschnitt weisen Geflüchtete, die als Arbeiterinnen oder Angestellte beschäftigt waren, neun Jahre Berufserfahrung auf. Zum Zeitpunkt der Befragung waren jedoch nur $14 \%$ der Befragten in Deutschland erwerbstätig. Da etwa $90 \%$ der Befragten angaben, dauerhaft in Deutschland bleiben zu wollen (von Gostomski et al. 2016, S. 6), scheinen vermehrte Bemühungen um eine gelungene Integration in die deutsche Gesellschaft und den deutschen Arbeitsmarkt erforderlich. Bislang üben Geflüchtete auch nach längerer Aufenthaltsdauer überwiegend einfache berufliche Tätigkeiten aus (Winther und Jordanoski 2016, S. 35) und dies trotz mittleren und hohen Bildungsniveaus. Nach vierjährigem Aufenthalt gehen entsprechend rund $65 \%$ dieser Gruppe einer Beschäftigung unterhalb ihres Bildungsniveaus nach (Fendel und Romiti 2015, S. 18).

Die geschilderte Lage der Geflüchteten ist mit Blick auf die Integration in den deutschen Arbeitsmarkt problematisch: Zum einen können vorhandene Kompetenzen oft nicht nachgewiesen werden, da aufgrund eines informellen oder non-forma-

\footnotetext{
1 Asylerstanträge bis November 2017.

2 Interviewt wurden erwachsene Geflüchtete, die von Januar 2013 bis Ende Januar 2016 in Deutschland eingereist sind und einen Asylantrag gestellt haben. Auf Basis des Ausländerzentralregisters wurde eine Zufallsstichprobe gezogen, wobei neben den gezogenen Personen auch deren Haushaltsmitglieder befragt wurden, sofern sie die Kriterien erfüllten (von Gostomski et al. 2016, S. 17f.).
} 
len Erwerbs keine formalen Nachweise wie Zeugnisse oder Zertifikate vorliegen. Die Vermittlung in eine Erwerbstätigkeit ist nur schwer möglich, da Arbeitgebern eine Entscheidungsgrundlage fehlt, wenn ausländische Abschlüsse nicht durch formale Nachweise belegt bzw. aufgrund fehlender Kenntnis ausländischer (Aus-)Bildungssysteme nicht eingeordnet werden können. In der Folge verbleiben viele Geflüchtete in Helfertätigkeiten und werden damit unterhalb ihres Qualifikationsniveaus eingesetzt. Für eine gelingende Integration in den deutschen Arbeitsmarkt ist es also notwendig, vorhandene fachliche Kompetenzen einerseits sichtbar und andererseits für den Arbeitsmarkt verwertbar zu machen.

In den letzten Jahren sind im Rahmen bildungspolitisch initiierter Projekte verschiedene Ansätze und Instrumente zur Kompetenzfeststellung entstanden, die versuchen non-formal und informell erworbene Kompetenzen zu erfassen. Dazu gehören beispielsweise das Verbundprojekt VALIKOM der Handwerks-, Industrie- und Handelskammern (Rehbold und Oehme 2017) oder umfangreiche Testbatterien, wie sie im Rahmen der Forschungsinitiative ASCOT entwickelt und beforscht wurden (Beck et al. 2016). Mit Blick auf die spezielle Zielgruppe der Geflüchteten weisen bestehende Verfahren jedoch einige Nachteile auf:

Sie sind erstens zeit- und/oder personalaufwändig, sodass ein flächendeckender Einsatz bei der großen Personengruppe der Geflüchteten kaum möglich scheint. Zweitens erfordert der Einsatz Sprachkenntnisse im Deutschen, die in der Regel erst nach längerem Aufenthalt in Deutschland vorhanden sind. Übersetzungen in verschiedene Sprachen liegen in der Regel nicht vor, was häufig den Einsatz von Dolmetschern erforderlich macht. Eine schnelle und effiziente Erfassung von informell oder non-formal erworbenen beruflichen Kompetenzen kann daher mit diesen Instrumenten (derzeit) oft nicht geleistet werden. Eine mögliche Lösung ist der Einsatz von zeiteffizienten papier- oder computerbasierten Testverfahren in verschiedenen Testsprachen und mit Bezug zu deutschen Referenzberufen. Anhand von deutschen Referenzberufen können fachliche Kompetenzen erfasst werden, die unter anderem durch Arbeitserfahrung, Erwerbstätigkeit oder in anderen Kontexten erworben wurden und möglicherweise für den Einsatz auf dem deutschen Arbeitsmarkt relevant sind.

Der vorliegende Beitrag befasst sich mit der Frage, wie solche berufsfachlichen Kompetenzen von Geflüchteten getestet werden können. Um einer Beantwortung dieser Frage näher zu kommen, wird anhand des Projekts IdA KompetenzCheck demonstriert, dass Kompetenztests für Geflüchtete nötig sind, und dass eine nachweisliche valide Kompetenzfeststellung über derartige Kompetenztests möglich ist. Auch jenseits der Zielgruppe Geflüchteter bieten Kompetenztests vielfältige Chancen für den Arbeits- und Weiterbildungsmarkt. Im vorliegenden Projekt wurden jedoch keine Erkenntnisse zu anderen Personengruppen generiert.

\section{Multimediale Testverfahren zur Feststellung fachlicher Kompetenzen}

In den letzten Jahren und Monaten sind neben einem klaren Problembewusstsein (Döring und Severing 2016) auch zunehmend Projekte zur Entwicklung von berufsfachlichen Kompetenzfeststellungsverfahren zu konstatieren: Das Spektrum reicht 
dabei von einfachen Selbsteinschätzungen (z.B. Erpenbeck und von Rosenstiel 2007) bis zu zeitaufwändigen Verfahren, die auf Simulationen oder der Kombination von verschiedenen Instrumenten wie Fachgesprächen, Bilanzierungsbögen und Arbeitsproben basieren (z. B. Oehme und Rehbold 2017; Nickolaus et al. 2011).

In Projekten wie dem IdA KompetenzCheck (s. unten) oder MYSKILLS: Berufliche Kompetenzen Erkennen (Döring et al. 2016; Herdin und Wink 2016) werden für ausgewählte Referenzberufe Testverfahren entwickelt, über die zeiteffizient große Personenkreise parallel getestet werden können. Den Testverfahren ist ein ähnlicher Ansatz gemeinsam: Es geht um eine valide Erfassung fachlicher Kompetenzen, die über eine reine Selbsteinschätzung hinausgeht und Rückschlüsse auf eine mögliche Handlungsfähigkeit in Bezug auf typische Handlungsfelder eines Referenzberufs zulässt.

Berufliche Kompetenzen sind kontextspezifische Leistungsdispositionen, die sich funktional auf Situationen und Anforderungen in beruflichen Kontexten beziehen. (Döring et al. 2016, S. 68; vgl. Klieme und Leutner 2006; Weinert 2001; Terzer et al. 2008)

Unter einem beruflichen Kontext lässt sich wiederum jeder abgrenzbare Bereich eines Berufsbildes fassen, der grundlegende Anforderungen widerspiegelt (Baethge und Seeber 2016). Mit Blick auf verbreitete Rahmenwerke zu Kompetenzen im beruflichen Kontext (KMK 2011; AK DQR 2011) lassen sich derart verstandene Kompetenzen konzeptuell als Bestandteil von Fachkompetenz ansehen, welche wiederum als definierender Bestandteil umfassender beruflicher Handlungskompetenz verstanden wird, im Sinne einer „Bereitschaft und Befähigung des Einzelnen, sich in beruflichen, gesellschaftlichen und privaten Situationen sachgerecht durchdacht sowie individuell und sozial verantwortlich zu verhalten“ (KMK 2011, S. 15).

Für die Messung von berufsbezogenen Kompetenzen empfehlen Baethge und Seeber (2016) den Rückgriff ,,auf berufstypische Arbeitshandlungen, -prozesse und -produkte, die weitgehend unabhängig von spezifischen betrieblichen Organisationsstrukturen und Arbeitsplatzbedingungen modelliert werden“ (ebd. S. 19f). Benötigt werden also Testverfahren, die typische Handlungssituationen von (Referenz-)Berufen in den Blick nehmen und diese mit Hilfe von Testaufgaben möglichst realitätsnah und authentisch abbilden.

Mit Blick auf moderne Kompetenztests lassen sich zwei Umsetzungsmöglichkeiten unterscheiden:

- Der erste Ansatz basiert auf komplexen (Computer-)Simulationen, in denen interaktiv bestimmte Aufgaben zu erfüllen sind (z. B. Walker et al. 2016; Fischer et al. 2015).

- Der zweite Ansatz - der in der vorliegenden Arbeit näher beleuchtet wird - basiert auf Fragen zu Arbeitshandlungen, -prozessen und -produkten, die in Hinblick auf eine Problemsituation zu beantworten sind, welche über Texte, Bilder und Videos

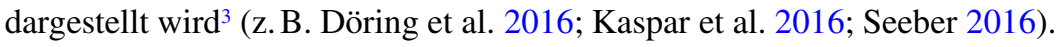

\footnotetext{
3 Am Rande sei erwähnt, dass die Ansätze sich nicht ausschließen und dass auch Mischformen durchaus verbreitet sind (z. B. Abele et al. 2016).
} 
In allen genannten Fällen wird Authentizität mit beruflichen Arbeitssituationen im Rahmen der Testverfahren angestrebt. Empirisch lassen sich wesentliche Anteile dessen, was sich über Performanz in Computersimulationen messen lässt, nachweislich auch über text-, bild- und/oder videobasierte Tests erfassen (so berichten z.B. Abele et al. (2016) für einen Test der Kompetenz von Kfz-Mechatronikern, dass simulationsbasierte Tests untereinander in derselben Größenordnung korrelieren wie ein simulationsbasierter Test mit einem inhaltlich ähnlichen videobasierten Test). Während computerbasierte Simulationen oft zeitaufwändig sind und daher vorwiegend in wissenschaftlichen Untersuchungen Verwendung finden, ist die Testdauer bei text-, bild- oder videobasierten Tests (im Folgenden TBV-Tests) in der Regel geringer, was eine zeitökonomische reliable Testung mit einer großen Anzahl unterschiedlicher Items erleichtert.

Dabei ist hervorzuheben, dass die Kompetenztestung über TBV-Tests in verschiedener Hinsicht über die Erfassung rein theoretischer Fachkenntnisse hinausgeht: Zum einen muss zur Lösung handlungsbezogener Aufgaben das erforderliche Fachwissen situationsadäquat anwendbar sein, was neben explizitem Wissen auch implizites Wissen erfordert, das in der Regel nicht einfach in Worte zu fassen ist. Dieser Zusammenhang wird durch die hohen Korrelationen mit simulationsbasierten Testverfahren unterstrichen. Das Einbringen impliziten Wissens in die Testung wird auch durch die Darstellungsweise von TBV-Tests ermöglicht: Die Darstellung von Handlungssituationen und Antwortoptionen erfolgt deshalb bei TBV-Tests nicht ausschließlich sprachlich (über Texte und ggf. Zahlenangaben), sondern auch visuell (über Bilder und Videos). Dadurch können Aufgaben teilweise auch durch implizites Wissen (z.B. das Wiedererkennen eines Werkzeugs, das in einer bestimmten Situation erforderlich ist) gelöst werden, ohne dass explizites Fachwissen verfügbar sein muss. Außerdem sind neben Kenntnissen auch kognitive Fertigkeiten wie Lesen und Rechnen für die Lösung mancher Aufgaben erforderlich (vgl. zu „kognitiven Fertigkeiten“ Anderson 1982). Motorische Fertigkeiten sind zwar in der Regel nicht direkt Teil der Testung (Döring et al. 2016, S. 23), allerdings ist für die richtige Lösung der Aufgaben teilweise eine Reflexion auf erforderliche manuelle Abläufe nötig, was eine entsprechende individuelle Erfahrung voraussetzt (vgl. Baethge und Seeber 2016).

\subsection{Testentwicklung}

In der Literatur existiert bisher keine allgemein anerkannte Systematik, an der man die Entwicklung von Kompetenztests entlang von Berufen und ihrer zugrunde liegenden Ausbildungsordnung ausrichten könnte und die gleichzeitig den Forderungen von Seeber und Baethge (s. oben) gerecht wird.

Für die hier betrachtete Testentwicklung wurde folgendes Vorgehen gewählt, das als Blaupause für beliebige berufliche Kontexte betrachtet werden kann und in Abb. 1 skizziert ist. 


\begin{tabular}{|c|c|c|}
\hline Arbeitsschritt & Beschreibung & Methodik \\
\hline $\begin{array}{l}\text { 1. Bestimmung eines } \\
\text { vorläufigen } \\
\text { Kompetenzmodells }\end{array}$ & $\begin{array}{l}\text { Auswahl geeigneter "Referenzberufe“ } \\
\text { und Bestimmung von Kompetenzen }\end{array}$ & $\begin{array}{l}\text { Kriterien gestützte Auswahl; } \\
\text { Expertenworkshop }\end{array}$ \\
\hline $\begin{array}{l}\text { 2. Bestimmung von } \\
\text { Einsatzfeldern }\end{array}$ & $\begin{array}{l}\text { Reale Einsatzgebiete, repräsentiert über } \\
\text { vollständige Arbeitsprozesse }\end{array}$ & Arbeitsprozessanalysen \\
\hline $\begin{array}{l}\text { 3. Ableitung relevanter } \\
\text { Handlungssituationen }\end{array}$ & $\begin{array}{l}\text { Beschreibung repräsentativer Situationer } \\
\text { im betrieblichen Kontext }\end{array}$ & $\begin{array}{l}\text { Abstimmung mit betrieblichen } \\
\text { Praxisexperten }\end{array}$ \\
\hline 4. curriculare Validierung & $\begin{array}{l}\text { Strukturen und Inhalte identifizieren } \\
\text { anhand von Ordnungsmitteln }\end{array}$ & $\begin{array}{l}\text { Analyse von Ausbildungsver- } \\
\text { ordnungen und Rahmenlehrplänen }\end{array}$ \\
\hline 5. ökologische Validierung & $\begin{array}{l}\text { Tätigkeiten beschreiben und durch } \\
\text { Experten validieren lassen }\end{array}$ & Expertenrating \\
\hline 6. Aufgabenentwicklung & $\begin{array}{l}\text { Aufgaben zu Handlungssituationen in } \\
\text { Zusammenarbeit mit Fachexperten } \\
\text { entwickeln }\end{array}$ & Expertenworkshop \\
\hline $\begin{array}{l}\text { 7. video- und bildbasierte } \\
\text { Umsetzung }\end{array}$ & $\begin{array}{l}\text { Produktion der Videos, Bild- und } \\
\text { Textgestaltung }\end{array}$ & Drehbuch/Skripte \\
\hline 8. Prüfung Testverfahren & $\begin{array}{l}\text { Überprüfung der Durchführbarkeit, } \\
\text { Verständnis, Akzeptanz und Eignung in } \\
\text { der relevanten Zielgruppe }\end{array}$ & $\begin{array}{l}\text { Feldtest, kognitiver Test (z.B. über } \\
\text { Think-aloud-Protokolle) }\end{array}$ \\
\hline 9. Itemselektion & $\begin{array}{l}\text { Überprüfung und ggf. Ausschluss } \\
\text { unpassender Items anhand geeigneter } \\
\text { Stichprobe (bis zur Einsatzreife) }\end{array}$ & $\begin{array}{l}\text { Kalibrierung anhand ausreichend } \\
\text { großer Stichprobe }\end{array}$ \\
\hline
\end{tabular}

Abb. 1 Allgemeine Prozessbeschreibung der Konstruktion von Kompetenztests

$\mathrm{Zu}$ Beginn werden die Einsatzfelder bestimmt, über die der Test eine Aussage treffen soll, ${ }^{4}$ d.h. es wird spezifiziert, (1) welche Kompetenzen gemessen werden sollen, (2) in welchen Einsatzfeldern sich diese Kompetenzen bei der Berufsausübung zeigen, und (3) welche konkreten Handlungssituationen in jedem Handlungsfeld zu bewältigen sind, um auf Kompetenz im Handlungsfeld zu schließen.

Die folgenden beiden Schritte des Konstruktionsprozesses befassen sich mit der Validierung und Erweiterung der bisherigen Überlegungen: Die identifizierten Handlungsdispositionen, -felder, und -situationen sind daraufhin zu prüfen, ob sie einerseits durch bestehende Ordnungsmittel wie Ausbildungsverordnungen und Rahmenlehrpläne abgedeckt werden (4) sowie andererseits der beruflichen Praxis gerecht werden (5). Das Resultat dieser Schritte ist ein konzeptuell vollständiges Kompetenzmodell, das alle unterscheidbaren Dimensionen (sog. Kompetenzstruktur) und

\footnotetext{
4 Zwischen den ersten drei Schritten herrscht ein hoher Grad an Abhängigkeit, weswegen z. B. Döring et al. (2016) sie unter einem einzelnen Arbeitsschritt „Bestimmung des Tätigkeitsfeldes“ subsummieren. Eine Antwort auf die Frage, welche Leistungen in welchen Situationen eine Leistungsdisposition indizieren, kann sich z. B. daraus ergeben, welche Handlungssituationen bzw. Handlungen ein Handlungsfeld in der Berufspraxis charakterisieren.
} 
alle curricular und praktisch relevanten bzw. unterscheidbaren Schwierigkeitsgrade (sog. Kompetenzniveaus) abdeckt (vgl. Klieme und Leutner 2006).

In einem nächsten Schritt werden (6) von Fachexperten Aufgaben entwickelt, die Rückschlüsse auf die oben bestimmten Leistungsdispositionen erlauben, und (7) die Aufgaben als text-, bild- oder videobasiertes Testverfahren umgesetzt. Im Anschluss wird eine mindestens zweistufige empirische Überprüfung des Testverfahrens durchgeführt (8). Qualitativ wird an Probanden der angezielten Zielgruppe das Testverfahren auf seine Funktionsfähigkeit, sachliche Korrektheit, Verständlichkeit und Unmissverständlichkeit der Aufgaben überprüft (9). Quantitativ wird das angedachte Messmodell an einer Stichprobe der Zielgruppe getestet, um die Güte des entwickelten Verfahrens festzustellen und bei Bedarf Revisionen an den zugrunde gelegten theoretischen Annahmen (bzw. dem verwendeten Messmodell) oder an den entwickelten Testaufgaben vorzunehmen.

Das skizzierte Vorgehen bildet die Grundlage für die hier vorgestellten Verfahren und bildet auch die Grundlage für die 2017 und 2018 entwickelten Testverfahren der Bundesagentur für Arbeit unter dem Titel MYSKILLS und wird im Folgenden näher erläutert.

\subsection{Besonderheiten bei der Zielgruppe Geflüchteter}

Bei der Entwicklung von Kompetenztests für die Zielgruppe der Geflüchteten sind einige Besonderheiten zu beachten. Zum einen sind aufgrund der Vielzahl an Geflüchteten zeiteffiziente Verfahren gefragt, an denen parallel eine Vielzahl an Personen teilnehmen können. Gleichzeitig sollen reliable und valide Aussagen über die Kompetenzen der Getesteten abgeleitet werden können (Döring und Severing 2016). Darüber hinaus sollten die Testergebnisse, um einen Nutzen für die Getesteten entfalten zu können, anschlussfähig in Bezug auf den deutschen Arbeitsmarkt sein (Hecker et al. 2019). Vor diesem Hintergrund liegt es nahe, computerbasierte Testverfahren einzusetzen, und die Gestaltung der Testaufgaben/-materialien an betriebliche Realitäten in Deutschland anzulehnen.

Ein zentrales Thema bei der Testung von Geflüchteten stellt die Testsprache dar. Ein kompetenter Umgang mit der deutschen Sprache stellt zwar in vielen Berufen eine Voraussetzung für erfolgreiche Arbeit dar, sollte aber nicht mit beruflicher Kompetenz vermengt werden. Um berufliche Kompetenz unabhängig von sprachlicher Kompetenz testen zu können, müssen Sprachbarrieren überwunden werden. Hierzu bietet es sich an, die Tests in den jeweiligen Herkunftssprachen anzubieten. Neben einer Übersetzung ins Englische und Arabische (um nur zwei Beispiele zu nennen) lassen sich auch durch einfache Sprache und die Einbindung multimedialer Darstellungen Sprachbarrieren reduzieren (Döring und Severing 2016). Besondere Schwierigkeiten sind zu bedenken, wenn Fachbegriffe übersetzt oder umschrieben werden müssen. So lautet eine mögliche Übersetzung des Fachbegriffs „Geogitter“ etwa (grob ,Gitter aus Kunststoff, das zur Stabilisierung des Untergrundes von Bauarbeiten dient“). Diese Übersetzung wäre jedoch problematisch im Kontext einer Aufgabe wie „Wozu dienen Geogitter?“. Auch um Differential Item Functioning (Koller et al. 2012) für verschiedene Testsprachen zu vermeiden, kann es sich anbieten, Fachbegriffe 
$\mathrm{zu}$ umschreiben. Umgekehrt kann es jedoch auch auftreten, dass bei gängigen Fachbegriffen eine Umschreibung zu Verwirrung und vermeidbarer Mehrdeutigkeit führt, sodass die Empfehlung nicht ohne Augenmaß umgesetzt werden sollte. Bei computerbasierten Tests ist überdies konfundierenden Effekten von Medienkompetenz entgegenzuwirken (z.B. durch einfache und intuitive Gestaltung - orientiert an bekannten Eingabeformaten wie gängigen Formularen - oder durch einführende Tutorials).

\subsection{Beispiel IdA KompetenzCheck im Bereich Metallbearbeitung und -verarbeitung}

Im Rahmen der Initiative Integration durch Ausbildung und Arbeit (IdA) der Vereinigung der Bayerischen Wirtschaft e. V. (vbw) wurden mit den IdA KompetenzChecks vom Forschungsinstitut Betriebliche Bildung (f-bb) Kompetenztests für ausgewählte Branchen (Metall, Elektro, Logistik und Garten-/Landschaftsbau) entwickelt, um berufliche Vorerfahrungen und Kompetenzen von Geflüchteten zu testen und eine erste Standortbestimmung vorzunehmen. Die Testkonstruktion folgte dabei im Wesentlichen dem oben vorgestellten Prozess der Testentwicklung (Abschn. 2.1) unter Berücksichtigung von Besonderheiten der Zielgruppe Geflüchteter (Abschn. 2.2).

Im Rahmen der IdA KompetenzChecks werden Personen in einer von vier Testsprachen (Deutsch, Englisch, Französisch oder Arabisch) mit Beschreibungen, Abbildungen und (in der computerbasierten Fassung) Videos realistischer Handlungssituationen konfrontiert und müssen darauf bezogene Fragen zu Handlungen und Prozessabläufen beantworten, die Rückschlüsse auf berufsfachliche Kompetenzen erlauben. Im Falle des IdA KompetenzChecks für den Bereich der Metallbearbeitung und -verarbeitung werden Kompetenzen in drei Handlungsfeldern gemessen: (1) Kompetenz beim Herstellen von Bauteilen und Baugruppen; (2) Kompetenz beim Einrüsten, Umrüsten und Bestücken von Maschinen und Anlagen, sowie (3) Kompetenz beim Prüfen und Messen in der Qualitätskontrolle. Diese drei Kompetenzen wurden in Anlehnung an das Teilqualifizierungsmodell für den Beruf des Maschinen- und Anlagenführers (Zeller, Neumann, Kohl, Krenn \& Küfner, 2011) sowie in enger Zusammenarbeit mit fachlichen Experten konzeptualisiert, decken das Berufsbild jedoch nicht vollständig ab.

Die Kompetenz in jedem Handlungsfeld wird beim IdA KompetenzCheck in Form eines separaten Teilergebnisses gemessen. Dem liegt ein einfaches und bzgl. seiner Annahmen sparsames Messmodell zugrunde: Wer schon einmal in einem Handlungsfeld gearbeitet hat, hat ipso facto Erfahrung im Umgang mit den zum Handlungsfeld gehörenden Handlungssituationen und weiß aus diesem Grund mit größerer Wahrscheinlichkeit die richtigen Antworten auf diesbezügliche handlungsbezogene Fragen. ${ }^{5}$ Auf Basis dieser Überlegungen wird aus den Antworten einer Person auf ihre Disposition zu Leistungen im Handlungsfeld geschlossen.

\footnotetext{
5 Einschränkend ist an dieser Stelle zu bemerken, dass manche Kompetenzen auch handlungsfeldübergreifend oder auf andere Weise erworben werden können und dass deren Messung und separate Ausweisung - sofern angestrebt - eine andere Herangehensweise oder komplexere Messmodelle erfordern kann (z. B. Fischer et al. 2015; Kaspar et al. 2016; Erpenbeck und Von Rosenstiel 2007).
} 


\section{Methode}

Auf Basis der bereits beschriebenen Vorgehensweise (siehe Abschn. 2.1) wurde unter Berücksichtigung der Besonderheiten Geflüchteter (siehe Abschn. 2.2) mit dem IdAKompetenzCheck im Bereich Metallbe- und -verarbeitung (siehe Abschn. 2.3) ein Testverfahren entwickelt, das die Feststellung von Kompetenzen in ausgewählten Handlungsfeldern dieser Branche unterstützen soll. Im Folgenden werden empirische Ergebnisse zu diesem Test berichtet, um zu überprüfen, ob auf die beschriebene Art und Weise berufliche Kompetenzen getestet werden können.

\subsection{Stichprobe}

Getestet wurden $n=162$ Geflüchtete im Rahmen des IdA-Projektes. Von den teilnehmenden Personen sind etwa $68 \%$ männlich und $11 \%$ weiblich $(21 \%$ fehlende Werte $\left.^{6}\right)$. Im Durchschnitt sind die Personen etwa 30 Jahre alt $(\mathrm{M}=32,2 ; \mathrm{SD}=7,6)$. Bzgl. der Testsprache bearbeiteten 59\% den Test in deutscher Sprache, 22\% in englischer Sprache und 19\% in arabischer Sprache. Berufserfahrung war - für den Fall, dass Berufserfahrung vorlag - in Jahren anzugeben. Fehlende Angaben waren entsprechend als 0 zu werten. $31 \%$ der Befragten erhielten einen Wert größer 0, die restlichen $69 \%$ einen Wert von 0. Im Median verfügten die Befragten mit Berufserfahrung über 3 Jahre Erfahrung.

\subsection{Datenerhebung und -aufbereitung}

Die Daten wurden im Rahmen von IdA 1000 in Integrationskursen zwischen März 2016 und April 2017 in Gruppentestungen erhoben. Die Teilnahme an einem der KompetenzChecks war freiwillig (wobei Berufserfahrung im jeweiligen Referenzberuf im Rahmen der Ausschreibung als wünschenswert dargestellt wurde, aber nicht zwingend erforderlich war). Die Teilnehmenden bearbeiteten unter Aufsicht von Kursleitung und je einem Projektmitarbeitenden eine papierbasierte Version des IdA KompetenzChecks. Als Anreiz wurde den Teilnehmenden eine vorläufige Rückmeldung über die festgestellten Kompetenzen zugestellt.

Jede unabhängige Antwort wurde binär als richtig oder falsch bewertet. Items mit extremer Schwierigkeit, die also von keiner Person oder von jeder Person gelöst werden konnten, wurden vor den folgenden Analysen entfernt (dies betraf lediglich ein Item in Testabschnitt 2, welches von keiner Person aus dem Kreis der Teilnehmenden richtig beantwortet wurde). Das Unterlassen einer Antwort wurde generell mit 0 bewertet.

\footnotetext{
${ }^{6}$ Das Geschlecht wurde nicht direkt erfragt, sondern lediglich anhand des Namens geschätzt. Mit dieser Fremdeinschätzung geht eine gewisse Unsicherheit einher, allerdings scheint uns diese Einschätzung informativer zu sein als der Verzicht auf eine Schätzung des Geschlechterverhältnisses. Zu fehlenden Werten kam es entsprechend, wenn der Name einer Person nicht mit ausreichender Sicherheit auf das Geschlecht schließen ließ $(n=34)$.
} 
Wright Map für alle Testabschnitte des IdA KompetenzCheck Metall

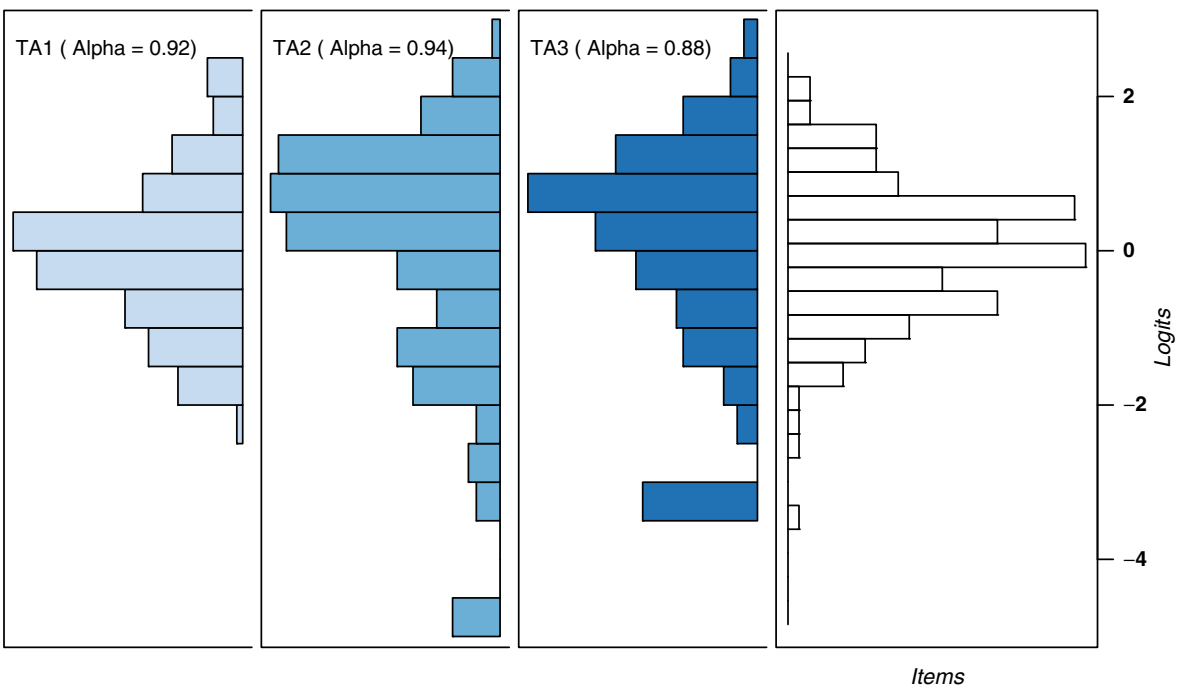

Abb. 2 Histogramm der Itemschwierigkeiten (rechts in weiß) und der Kompetenz in jedem der drei Testabschnitten (TA, links in dunkleren Farbtönen)

\subsection{Auswertung}

Zur Auswertung wurde das herkömmliche Rasch-Modell angewendet, welches den Summenwert über alle binär bewerteten Items als suffiziente Statistik zur Schätzung des zu messenden Personenparameters annimmt. Die Schwierigkeit der Items wurde, wie bei der Rasch-Modellierung üblich, für jeden Testbereich auf eine Summe von 0 normiert (Summe-Null-Normierung, Bühner 2005) wodurch die Personenparameter im Verhältnis zur Itemschwierigkeiten kriteriumsorientiert interpretiert werden können.

Von einem komplexeren zweiparametrischen logistischen (2PL) Modell wird abgesehen, weil dieses durch eine Verdopplung der zu schätzenden Parameter eine Verringerung der Schätzgenauigkeit impliziert hätte und weil im 2PL-Modell bei der Bestimmung des Personenparameters Items mit hoher Trennschärfe mehr wiegen als Items mit niedriger Trennschärfe (unabhängig von der Item-Schwierigkeit), selbst wenn theoretisch alle Items als gleichermaßen wichtig für kompetentes Verhalten erachtet werden (Robitzsch 2009). Auch eine Beurteilung der absoluten Ausprägungen von Modell- und Itemfit unterbleibt an dieser Stelle aufgrund der für diesen Zweck unzureichend großen Stichprobe von unter 500 Teilnehmenden (Stone und Yumoto 2004). Die Analysen der vorliegenden Arbeit basieren demgegenüber auf Schätzwerten der Personen- bzw. Itemparameter. Diese können den Analysen von Stone und Yumoto (2004) zufolge bereits ab 100 Teilnehmenden mit ausreichender Genauigkeit geschätzt werden. 


\section{Ergebnisse}

Kompetenzen sind bekannt als verhältnismäßig heterogene Konstrukte (vgl. Fischer und Neubert 2015). Dennoch ist die Reliabilität des vorliegenden Tests für alle Testabschnitte aufgrund einer ausreichend großen Itemzahl als gut zu bewerten $(\alpha>0,87$, siehe Abb. 2) und die Trennschärfe aller Items ist größer als 0 .

Abb. 2 zeigt die Itemschwierigkeit (Itemparameter) der verwendeten Items (Abbildung rechts, in weiß dargestellt) und stellt diese den Kompetenzausprägungen (Personenparametern) der getesteten Personen (Abbildung links, in dunkleren Farbtönen dargestellt) gegenüber. Wie die Abbildung verdeutlicht, entspricht der Durchschnitt der Personenparameter für alle drei Testabschnitte in etwa dem Durchschnitt der normierten Itemparameter.

Effekte der Berufserfahrung wurden für jeden Testabschnitt über einen t-Test bestimmt, in den die dichotomisierte Berufserfahrung (Berufsjahre $>0$ ) als unabhängige Variable einging und der Personenparameter als abhängige Variable. Signifikante Effekte von Berufserfahrung auf die Leistungen im jeweiligen Testabschnitt finden sich in allen drei Testbereichen (TA1: $t=3,14 ; d f=77,77 ; p<0,01$; TA2: $t=2,74$; $d f=107,45 ; p<0,01 ;$ TA3: $t=2,82 ; d f=104,11 ; p<0,01)$. Dies kann als Beleg für die Behauptung verstanden werden, dass mit den entwickelten Aufgaben in der Tat durch Arbeitserfahrung erworbene Fachkompetenzen gemessen werden.

\section{Diskussion}

Mit der vorliegenden Arbeit legen wir eine Systematik vor, anhand derer Kompetenztests für unterschiedliche berufliche Kontexte entwickelt werden können (siehe Abschn. 2.1) und demonstrieren anhand von Daten zum IdA KompetenzCheck im Bereich Metallbe- und -verarbeitung die Reliabilität und Validität des entwickelten Testverfahrens. Wie unsere Ergebnisse zeigen, scheint es möglich, fachliche Kompetenzen auf reliable und valide Weise zeiteffizient zu erfassen. Die Aufgaben wurden sorgfältig ökologisch und curricular validiert, und die erwartungsgemäßen Effekte von Berufserfahrung auf die Kompetenzen im oben berichteten Test bieten einen empirischen Anhaltspunkt dafür, dass auf diese Weise ausgewählte fachliche Kompetenzen bei Geflüchteten effektiv und effizient gemessen werden können.

Der IdA KompetenzCheck liefert damit einen wertvollen Beitrag zur Validierung von Lernergebnissen nicht-zertifizierten non-formalen und informellen Lernens. Allerdings ist hervorzuheben, dass der IdA KompetenzCheck aus Gründen der Praktikabilität nicht das Ziel hat, berufliche Handlungskompetenz in ihrer vollen Breite zu erfassen. Aspekte wie Sprachfertigkeit, Sozial- und Selbstkompetenz sind nicht Gegenstand der Testung, und auch die getesteten Fachkompetenzen decken das Berufsbild nicht vollständig ab. Der IdA KompetenzCheck wurde als Testverfahren entwickelt, um einen ersten Anhaltspunkt über die Kompetenzen in unterschiedlichen berufsrelevanten Handlungsfeldern zu gewinnen. Als solches bietet er vor allem für Personen ohne klassische Qualifikationen und Kompetenznachweise eine Möglichkeit, das Vorhandensein von Kompetenzen zu belegen. Im Gegensatz zu herkömmlichen Prüfungen reduziert der IdA KompetenzCheck durch die vorliegenden 
Testsprachen (Deutsch, Englisch, Arabisch und Französisch) und die Einbindung von Bildern und (in der computerbasierten Version) Videos von Handlungssituationen die Sprachbarriere. Gleichzeitig bietet er - im Gegensatz zu vielen anderen praxisnahen Verfahren, wie z. B. Arbeitsproben - aufgrund seiner hochgradig standardisierten Präsentation Ergebnisse, die über verschiedene Teilnehmende hinweg vergleichbar sind. Als wissenschaftlich entwickeltes Verfahren bietet er den Vorteil (gegenüber manchem ausländischen Abschlusszeugnis von zertifizierenden Stellen unbekannter Herkunft), dass empirische Belege seiner Gültigkeit vorliegen, die von Interessierten vor einer Interpretation der Ergebnisse herangezogen werden können. Die enge Anbindung der Ergebnisrückmeldung an bestehende Teilqualifizierungsmodelle wiederum fördert die Anschlussförderlichkeit der Testergebnisse. Unter der Annahme, dass die Testinhalte des IdA KompetenzCheck Metall die beruflichen Anforderungen adäquat repräsentieren, lässt sich beispielsweise feststellen, dass die getestete Stichprobe eine durchschnittliche Kompetenz in allen getesteten Handlungsfeldern aufweist, dass die getesteten Kompetenzen mit Berufserfahrung einher zu gehen scheinen und dass sich die Kompetenz einzelner Getesteter in jedem Handlungsfeld mit einer hohen Reliabilität bestimmen lässt.

Generell sind neben den Vorzügen des IdA KompetenzCheck jedoch auch seine Grenzen anzuerkennen: Der IdA KompetenzCheck wird z. B. ausschließlich auf Papier oder am Computer bearbeitet und erfordert kein praktisches Tätigwerden. Auch wenn die gestellten Fragen in gewissem Maße durchaus Rückschlüsse auf berufliche Erfahrung erlauben (vgl. hierzu die Ausführungen in Abschn. 2 und die empirischen Validitätsbelege in Abschn. 4) scheint es angebracht, den IdA KompetenzCheck um weitere Verfahren zu ergänzen (z.B. Arbeitsproben), um ein ganzheitliches Bild von den Kompetenzen einer Person zu gewinnen, da motorische Fertigkeiten nicht direkt Gegenstand der Testung sind.

Auch der Forschungsstand zum IdA KompetenzCheck und ähnlichen Verfahren muss zum jetzigen Zeitpunkt als vorläufig beurteilt werden: Die empirische Erprobung der psychometrischen Qualität von Tests, die gemäß der hier vorgeschlagenen Systematik entwickelt wurden, befindet sich aktuell noch in vollem Gange und eine umfassende Kriteriums- und Konstruktvalidierung steht noch aus. Auch ein empirischer Nachweis für den Nutzen des IdA KompetenzCheck für die betriebliche Praxis, bzw. für die Zielgruppe Geflüchteter, steht zurzeit noch aus. Die vorliegende Arbeit versteht sich insofern lediglich als erster Anhaltspunkt für die Praktikabilität, Reliabilität und Validität des vorgestellten Verfahrens zur Testentwicklung. Ein abschließendes Urteil scheint zum jetzigen Zeitpunkt jedoch weder angemessen noch sinnvoll.

Weitere Forschung bietet sich insbesondere in Bezug auf Subgruppenunterschiede oder komplexere Messmodelle an, die sich in Bezug auf die Heterogenität der Zielgruppe empfehlen könnten (z.B. die Modellierung latenter Klassen, vgl. Rost 1990), sobald eine ausreichend umfassende Datengrundlage vorliegt. Borsboom und Mellenbergh (2007, S. 98) bemerken ergänzend dazu, wie unpassend das traditionelle reflektive Messmodell - also ein Messmodell, welches zur Annahme hat, dass eine höhere Ausprägung des latenten Konstrukts zur Konsequenz hat, bzw. sich darin widerspiegelt/reflektiert, dass Testaufgaben mit einer höheren Wahrscheinlich- 
keit gelöst werden können (Fischer und Neubert 2015) - bei der Vermessung von Wissensbeständen erscheint:

the proportion of facts you know does not make a difference for whether you can answer a particular question (you either can or cannot, which depends on whether you know the answer), but this proportion does make a difference to the number of questions you can answer (assuming that they are a representative sample from the domain).

Überträgt man diese Überlegungen von Borsboom und Mellenbergh (2007) auf den Bereich der Kompetenztestung, bleibt festzustellen, dass ein Kompetenztest durchaus Validität aufweisen kann (also messen kann, was er zu messen vorgibt), ohne über eine ausgeprägte Reliabilität zu verfügen (also ohne dass die Antworten auf einzelne Items des Tests korrelieren müssten; vgl. auch Fischer und Neubert 2015). Ein großer Anteil richtig gelöster Aufgaben lässt in diesem Fall also auf Kompetenz schließen, auch wenn die Ausprägung der Kompetenz sich nicht kausal und gleichermaßen auf die Beantwortung aller Items auswirken muss. Dabei ist anzumerken - und durchaus im Einklang mit den vorgelegten empirischen Ergebnissen - dass sich Validität und Reliabilität durchaus nicht ausschließen müssen. Wie Fischer und Neubert (2015) erörtern kann es gerade bei breit angelegten Kompetenzen, die eine heterogene Menge an Anforderungen umfassen, für eine reliable und valide Messung erforderlich sein, mehrere Komponenten von Kompetenz separat zu messen (also z. B. getrennt nach homogenen Handlungsfeldern, wie im IdA KompetenzCheck erfolgt).

Die separate Messung mehrerer berufsrelevanter Kompetenzen trägt dabei nicht nur den o.g. messtheoretischen Erwägungen Rechnung, sondern bietet auch einen praktischen Mehrwert für Geflüchtete und Betriebe: Dadurch, dass der IdA KompetenzCheck Testergebnisse auf der Ebene von berufsrelevanten Handlungsfeldern ausweist, erhalten die Getesteten die Möglichkeit, vorliegende Kompetenzen auch dann differenziert zu belegen, wenn eine umfassende berufliche Handlungsfähigkeit noch nicht vorliegt. Sowohl die Getesteten als auch Betriebe, erhalten auf diese Weise einen aussagekräftigen Anhaltspunkt dafür, in welchen Handlungsfeldern fachliche Kompetenzen vorliegen und in welchen Handlungsfeldern ggf. Weiterbildungsbedarf besteht. Im besten Fall eröffnet die differenzierte Ergebnisrückmeldung des IdA KompetenzCheck die Möglichkeit, Personen einerseits zielführend zu fördern und andererseits passgenau den Stellen zuzuweisen, für die sie sich aufgrund der vorliegenden Kompetenzen am besten eignen. Auf Basis bisheriger Erfahrungen mit der Zielgruppe der Geflüchteten ist zu erwarten, dass weitere Qualifizierung vor einem beruflichen Einsatz oftmals notwendig ist - nicht nur vor dem Hintergrund fehlender Kenntnis der deutschen (Fach-)Sprache. Je konkreter die Tests Aussagen in Bezug auf die Einsatzfähigkeit am Arbeitsmarkt machen, desto zielgerichteter können fehlende Fertigkeiten nachqualifiziert werden, um bestehende Lücken gezielt zu schließen. Die bedarfsorientierte Nachqualifizierung kann teilweise auf Basis von Anpassungsqualifizierungen erfolgen. Jedoch erlangen Geflüchtete so keinen in Deutschland anerkannten Berufsabschluss. Alternativ können Berufsanschlussfähige Teilqualifikationen (TQ) zum Einsatz kommen, wie sie von der Bundesagentur für Arbeit gefördert werden. Teilqualifikationen sind Qualifizierungsbausteine, die sys- 
tematisch in Teilschritten auf einen Berufsabschluss vorbereiten (z. B. Zeller et al. 2011). Alle Teile zusammen ergeben einen vollständigen Beruf. Bei hoher Passung zwischen Teilqualifizierung und Handlungsfeldern des Testverfahrens besteht so die Möglichkeit genau die noch fehlenden aber für einen Beruf notwendigen Kompetenzen zu erwerben. Über die Externenprüfung besteht dann die Möglichkeit, einen formalen Berufsabschluss zu erreichen.

Angesichts der schwierigen Lage vieler Geflüchteter auf dem deutschen Arbeitsmarkt, und dem vielerorts beklagten Fachkräftemangel, bieten Verfahren wie der IdA KompetenzCheck sowohl für Geflüchtete als auch für deutsche Betriebe eine wertvolle Chance, vorhandenes Potential nutzbar zu machen.

Vor diesem Hintergrund möchten wir anregen, den IdA KompetenzCheck (ebenso wie zahlreiche andere Verfahren der Kompetenzfeststellung) weder als vollumfängliches Diagnostikum beruflicher Handlungskompetenz misszuverstehen, noch ihn aufgrund der o.g. Grenzen zu verwerfen. Wir halten den IdA KompetenzCheck vielmehr für einen gelungenen Beitrag zu einer Validierung von Kompetenzen, die im Rahmen von nicht-zertifiziertem, non-formalem und informellem Lernen erworben wurden, der jedoch im Sinne einer ganzheitlichen Beurteilung von beruflicher Handlungskompetenz (KMK 2011) andere Verfahren der Kompetenzfeststellung nicht ersetzen, sondern nur ergänzen kann.

Grundsätzlich sollte jedwede Erfassung rein fachlicher Kompetenzen zum Zwecke einer ganzheitlichen Beurteilung stets ergänzt werden (Fischer und Neubert 2015) um weitere Aspekte einer umfassend verstandenen beruflichen Handlungskompetenz (KMK 2011).

Open Access Dieser Artikel wird unter der Creative Commons Namensnennung 4.0 International Lizenz (http://creativecommons.org/licenses/by/4.0/deed.de) veröffentlicht, welche die Nutzung, Vervielfältigung, Bearbeitung, Verbreitung und Wiedergabe in jeglichem Medium und Format erlaubt, sofern Sie den/die ursprünglichen Autor(en) und die Quelle ordnungsgemäß nennen, einen Link zur Creative Commons Lizenz beifügen und angeben, ob Änderungen vorgenommen wurden.

Publisher's Note Springer Nature remains neutral with regard to jurisdictional claims in published maps and institutional affiliations.

\section{Literatur}

Abele, S., Behrendt, S., Weber, W., \& Nickolaus, R. (2016). Berufsfachliche Kompetenzen von Kfz-Mechatronikern von drei separa Kompetenzdimensionen und erzielte Leistungen (KOKO Kfz). In K. Beck, M. Landenberger \& F. Oser (Hrsg.), Technologiebasierte Kompetenzmessung in der beruflichen Bildung (S. 171-204). Bielefeld: W. Bertelsmann.

AK DQR/Arbeitskreis Deutscher Qualifikationsrahmen (2011). Deutscher Qualifikationsrahmen für lebenslanges Lernen. https://www.dqr.de/media/content/Der_Deutsche_Qualifikationsrahmen_fue_ lebenslanges_Lernen.pdf. Zugegriffen: 28. Mai 2018.

Anderson, J. R. (1982). Acquisition of cognitive skill. Psychological review, 89(4), 369-406

Baethge, M., \& Seeber, S. (2016). Die gemeinsame theoretische und methodische Basis der ASCOTProjekte. In K. Beck, M. Landenberger \& F. Oser (Hrsg.), Technologiebasierte Kompetenzmessung in der beruflichen Bildung - Resultate aus dem Forschungsprogramm ASCOT (S. 15-42). Bielefeld: W. Bertelsmann.

BAMF/Bundesamt für Migration und Flüchtlinge (2016). Aktuelle Zahlen zu Asyl. Ausgabe 12/2016. http://www.bamf.de/DE/Infothek/Statistiken/Asylzahlen/AktuelleZahlen/aktuelle-zahlen-asyl-node. html. Zugegriffen: 4. Jan. 2018. 
BAMF/Bundesamt für Migration und Flüchtlinge (2017). Aktuelle Zahlen zu Asyl. Ausgabe 11/2017. http://www.bamf.de/DE/Infothek/Statistiken/Asylzahlen/AktuelleZahlen/aktuelle-zahlen-asyl-node. html. Zugegriffen: 4. Jan. 2018.

Beck, K., Landenberger, M., \& Oser, F. (2016). Technologiebasierte Kompetenzmessung in der beruflichen Bildung. Ergebnisse aus der BMBF-Förderinitiative ASCOT. Bielefeld: W. Bertelsmann.

Borsboom, D., \& Mellenbergh, G. J. (2007). Test validity in cognitive assessment. In Cognitive diagnostic assessment for education: theory and applications (S. 85-118).

Brücker, H., et al. (2016). IAB-Kurzbericht 24/2016. Aktuelle Analysen aus dem Institut für Arbeitsmarktund Berufsforschung. http://doku.iab.de/kurzber/2016/kb2416.pdf. Zugegriffen: 28. Mai 2018.

Bühner, M. (2005). Einführung in die Test- und Fragebogenkonstruktion. Bd. 2. München: Pearson.

Döring, O., \& Severing, E. (2016). Anmerkungen zur Erfassung der beruflichen Kompetenzen von Flüchtlingen. Berufsbildung, 158, 34-36.

Döring, O., Neumann, F., Hecker, K., \& Fischer, A. (2016). Qualitätsstandards für Kompetenztests - Anforderungskatalog. https://www.f-bb.de/fileadmin/user_upload/Qualitaetsstandards_Kompetenztests_ Anforderungskatalog.pdf. Zugegriffen: 28. Mai 2018.

Erpenbeck, J., \& Von Rosenstiel, L. (2007). Handbuch Kompetenzmessung: Erkennen, verstehen und bewerten von Kompetenzen in der betrieblichen, pädagogischen und psychologischen Praxis (2. Aufl.). Stuttgart: Schäffer-Poeschel.

Fendel, T., \& Romiti, A. (2015). Die Bedeutung von Bildung und Spracherwerb für die Arbeitsmarktintegration von Asylsuchenden. Berufsbildung in Wissenschaft und Praxis, 45(1), 16-19.

Fischer, A., \& Neubert, J.C. (2015). The multiple faces of complex problems: a model of problem solving competency and its implications for training and assessment. Journal of Dynamic Decision Making, 1(1), 1-13. https://journals.ub.uni-heidelberg.de/index.php/jddm/article/view/23945

Fischer, A., Greiff, S., Wüstenberg, S., Fleischer, J., Buchwald, F., \& Funke, J. (2015). Assessing analytic and interactive aspects of problem solving competency. Learning and Individual Differences, 39, $172-179$.

von Gostomski, et al. (2016). IAB Forschungsbericht 14/2016. Aktuelle Ergebnisse aus der Projektarbeit des Instituts für Arbeitsmarkt- und Berufsforschung. http://doku.iab.de/forschungsbericht/2016/ fb1416.pdf. Zugegriffen: 28. Mai 2018.

Hecker, K., Schley, T., Fischer, A., Wittig, W., \& Pfeiffer, I. (2019). Erfassung beruflicher Kompetenzen von Migrantinnen und Migranten sowie Geflüchteten - Kriterien und Vorgehen bei der Konstruktion eines computerbasierten Kompetenztests. In A. David, et al. (Hrsg.), Migration und Arbeit (Revisited): Herausforderungen, Problemlagen und Gestaltungsinstrumente. Leverkusen: Budrich.

Herdin, G., \& Wink, R. (2016). Kompetenzen von Flüchtlingen erfassen - Anforderungen und Gestaltung von innovativen Kompetenzfeststellungsverfahren. In V. Heyse, J. Erpenbeck \& S. Ortmann (Hrsg.), Intelligente Integration von Flüchtlingen und Migranten (S. 103-112). Münster: Waxmann.

Kaspar, R., Döring, O., Wittmann, E., Hartig, J., Weyland, U., Nauerth, A., Möllers, M., Rechenbach, S., Simon, J., \& Worofka, I. (2016). Competencies in geriatric nursing: empirical evidence from a computer-based large-scale assessment calibration study. Vocations and Learning, 9(2), 185-206.

Klieme, E., \& Leutner, D. (2006). Kompetenzmodelle zur Erfassung individueller Lernergebnisse und zur Bilanzierung von Bildungsprozessen. Beschreibung eines neu eingerichteten Schwerpunktprogramms der DFG. Zeitschrift für Pädagogik, 52(6), 876-903.

KMK/Kultusministerkonferenz (2011). Handreichung für die Erarbeitung von Rahmenlehrplänen der Kultusministerkonferenz für den berufsbezogenen Unterricht in der Berufsschule und ihre Abstimmung mit Ausbildungsordnungen des Bundes für anerkannte Ausbildungsberufe. Bonn: KMK.

Koller, I., Alexandrowicz, R., \& Hatzinger, R. (2012). Das Rasch Modell in der Praxis. Eine Einführung mit $e$ Rm. Wien: Facultas.

Nickolaus, R., Abele, S., \& Gschwendtner, T. (2011). Prüfungsvarianten und ihre Güte: Simulationen beruflicher Anforderungen als Alternative zu bisherigen Prüfungsformen? In E. Severing \& R. Weiß (Hrsg.), Prüfungen und Zertifizierungen in der beruflichen Bildung (S. 83-95). Bielefeld: W. Bertelsmann.

Oehme, A., \& Rehbold, R. R. (2017). Abschlussbezogene Validierung non-formal und informell erworbener Kompetenzen (Verfahrensprototyp). Vortrag im Rahmen der Hochschultage Berufliche Bildung 2017 an der Universität zu Köln am 15. März 2017. http://www.fbh.uni-koeln.de/vortraege?page=1. Zugegriffen: 28. Mai 2018.

Rehbold, R.R., \& Oehme, A. (2017). Abschlussbezogene Validierung nonformal und informell erworbener Kompetenzen (ValiKom) - Konzeptionelle Überlegungen für die Entwicklung eines Verfahrensprototyps. https://www.berufsbildung.nrw.de/cms/upload/hochschultage-bk/2017beitraege/WS_ 07_Validierung_informeller_Kompetenzen_Rehbold_Oehme.pdf. Zugegriffen: 25. Mai 2018. 
Robitzsch, A. (2009). Methodische Herausforderungen bei der Kalibrierung von Leistungstests. In A. Bremerich-Vos, D. Granzer \& O. Koller (Hrsg.), Bildungsstandards Deutsch und Mathematik (S. 42-106). Weinheim: Beltz.

Rost, J. (1990). Rasch models in latent classes: an integration of two approaches to item analysis. Applied Psychological Measurement, 14(3), 271-282.

Seeber, S. (2016). Vom Domänenmodell zum Kompetenzmodell: Konturen eines Assessmentdesigns zur Messung beruflicher Fachkompetenzen bei Medizinischen Fachangestellten. http://www.bwpat.de/ profil4/seeber_profil4.pdf. Zugegriffen: 28. Mai 2018.

Stone, M., \& Yumoto, F. (2004). The effect of sample size on Rasch/IRT parameters using dichotomous items. Journal of applied measurement, 5, 48-61.

Terzer, E., Hartig, J., \& Upmeier zu Belzen, A. (2008). Systematische Konstruktion eines Tests zu Modellkompetenz im Biologieunterricht unter Berücksichtigung von Gütekriterien. Zeitschrift für Didaktik der Naturwissenschaften, 19, 51-76.

Walker, F., Link, N., \& Nickolaus, R. (2016). A multidimensional structure of domain-specific problemsolving competencies of electronics technicians for automation technology. Empirical Research in Vocational Education and Training, 8(1), 1-16.

Weinert, F.E. (2001). Vergleichende Leistungsmessung in Schulen - eine umstrittene Selbstverständlichkeit. In Leistungsmessungen in Schulen (S. 17-32). Weinheim: Beltz.

Winther, \& Jordanoski (2016). Was wir haben und was wir brauchen: Kompetenzanerkennung bei Flüchtlingen. DIE Zeitschrift für Erwachsenenbildung, 4, 34-36.

Zeller, B., Neumann, F., Kohl, M., Krenn, S. \& Küfner C. (2011). Schritt für Schritt zum/zur Maschinenund Anlagenfïhrer/-in: Zertifizierte Teilqualifikationen der Bundesagentur für Arbeit (Leitfaden für die Bildungspraxis). Bielefeld: wbv. 